\title{
Management of Multiple Endocrine Neoplasia Type 2A (MEN 2A): Diagnostic and Therapeutic Concerns with the First Documented Senegalese Family
}

\author{
Abdoulaye Leye ${ }^{1 *}$, Ngone Diaba Diack ${ }^{1}$, Michel Assane Ndour ${ }^{1}$, Nafy Ndiaye ${ }^{1}$, \\ Yakham Mohamed Leye ${ }^{1}$, Biram Codou Fall ${ }^{1}$, Malick Ndiaye ${ }^{2}$, Abdoul Karim Daher ${ }^{1}$, \\ Ahmed Tall Lemrabott ${ }^{3}$, Boucar Diouf ${ }^{3}$
}

${ }^{1}$ Internal Medicine/Endocrinology department. Teaching Hospital of Pikine/Dakar

${ }^{2}$ ENT Department. Fann Teaching Hospital/Dakar

${ }^{3}$ Nephrology Department. Le Dantec Teaching Hospital

Email: *ablayleye@hotmail.com

How to cite this paper: Leye, A., Diack, N.D., Ndour, M.A., Ndiaye, N., Leye, Y.M., Fall, B.C., Ndiaye, M., Daher, A.K., Lemrabott, A.T. and Diouf, B. (2018) Management of Multiple Endocrine Neoplasia Type 2A (MEN 2A): Diagnostic and Therapeutic Concerns with the First Documented Senegalese Family. Open Journal of Endocrine and Metabolic Diseases, 8, 29-37. https://doi.org/10.4236/ojemd.2018.81004

Received: December 31, 2017

Accepted: January 14, 2018

Published: January 17, 2018

Copyright (c) 2018 by authors and Scientific Research Publishing Inc. This work is licensed under the Creative Commons Attribution International License (CC BY 4.0).

http://creativecommons.org/licenses/by/4.0/

\section{(c) (i) Open Access}

\begin{abstract}
Introduction: Multiple endocrine neoplasia (MEN) type 2A is a multiglandular tumor condition inherited in an autosomal dominant manner. It is related to proto-oncogene RET mutation whose analysis is the best technique for family screening. It features in a variable way medullary thyroid cancer (MTC), primary hyperparathyroidism (HPT) and pheochromocytoma. The revealing manifestations of these tumors are often neglected for a long time and the screening should be systematic particularly in a known family context. Methods: After a family tree establishment of a MEN 2A index case, a family survey allowed to diagnose other cases in the family by means of biological, radiological and/or genetic examinations. Results: We report a family form of MEN 2A in a family of three households. In this family 13 people (index case included) were probed out of 34 members. The average age of our patients was 43.54 . The sex ratio men/women was 0.85 . The simultaneous diagnosis of a primary HPT and a MTC was carried out in our index case and constituted the circumstance of discovery of MEN 2A. The time limit of MEN 2A diagnosis on the other family members was on average 7.7 years. A MTC was recorded in 7 patients. It was asymptomatic in overall cases. A pheochromocytoma was present in only one patient. Primary HPT was found in four patients. Renal lithiasis with recurrent unilateral or bilateral nephritic colic attacks was the main manifestation. Besides the index case, 11 patients had a genetic testing. In 7 patients, a mutation on proto-oncogene RET located on
\end{abstract}


the codon 634 was noted. A surgical care was carried out on 6 patients. We recorded three patients lost to follow-up. A patient died before surgery. In the index case, biological and radiological monitoring found a locoregional residual disease that indicated surgical revision and radiotherapy. Prophylactic thyroidectomy was not performed in any case driven by lack of compliance and/or low income. Conclusion: The discovery of a MEN 2A case imposes genetic survey allowing the screening of other cases in the family and the establishment of a preventive strategy.

\section{Keywords}

Multiple Endocrine Neoplasia, Pedigree, RET Gene Senegal

\section{Introduction}

Multiple endocrine neoplasia (MEN) represents a group of conditions that associate many endocrine tumors. They occur simultaneously or successively on a same patient in a sporadic mode or fit in a frame of family forms [1] [2]. The type 2A of MEN formerly named Sipple Syndrome is the most common type. It's made by the association of Medullary Thyroid Cancer (MTC), pheochromocytoma and primary hyperparathyroidism (primary HPT) [3]. It is an autosomal dominant inherited condition. There are relatively rare conditions that impose a family survey since the diagnosis so that to detect beginning forms and to establish a preventive strategy.

We report cases of MEN 2A focusing on investigation of a Senegalese family following an index case diagnosis in Dakar [4].

\section{Methods}

Our study focused on a Senegalese family, including first-degree relatives (siblings and children) of an index case diagnosed with MEN 2A at the Internal Medicine department of Le Dantec Teaching Hospital in 1997.

It was about a descriptive cross-sectional study based on the working on the follow-up medical records of the polygamous family members, followed from 1997 to nowadays. The screening based on the research of proto-oncogene RET mutation carried out after an informed consent, and realised in France. The genetic diagnosis was retained after confirming the first results by a second sample. Biological examinations such as the dosage of calcitonin, urinary or plasma methoxyamine, parathyroid hormone and ionized calcium were performed also in France by sending blood sample via DHL Company. Morphological explorations such as scintigraphy and PET-Scan were realized in Morocco after patient transfer of the index case. The diagnosis of MEN 2Ain the family members of the index case was retained in the presence of clinical, biological and imaging signs suggesting CMT, pheochromocytoma or primary HPT, and detected mutation on proto-oncogene RET. 


\section{Results}

\section{Epidemiological description of the studied family}

The family consisted of a sibling of 22 people and 12 children and grandchildren (Figure 1, Figure 2, Figure 3) involving 34 people overall. There were three households. The index case came from the second household (Figure 1). The first and the third household were less explored (Figure 2, Figure 3). We had exploitable data on 13 people.

The average age of our patients was 43.5 years with extremes of 21 and 63 years. Nine of our patients were less than 40 years (Table 1 ).

The average age of the patients while the diagnosis was 31.5 years with extremes from 7 to 59 years. Our index case was 37 years at the time of diagnosis. The sex ratio men/women was 0.85 .

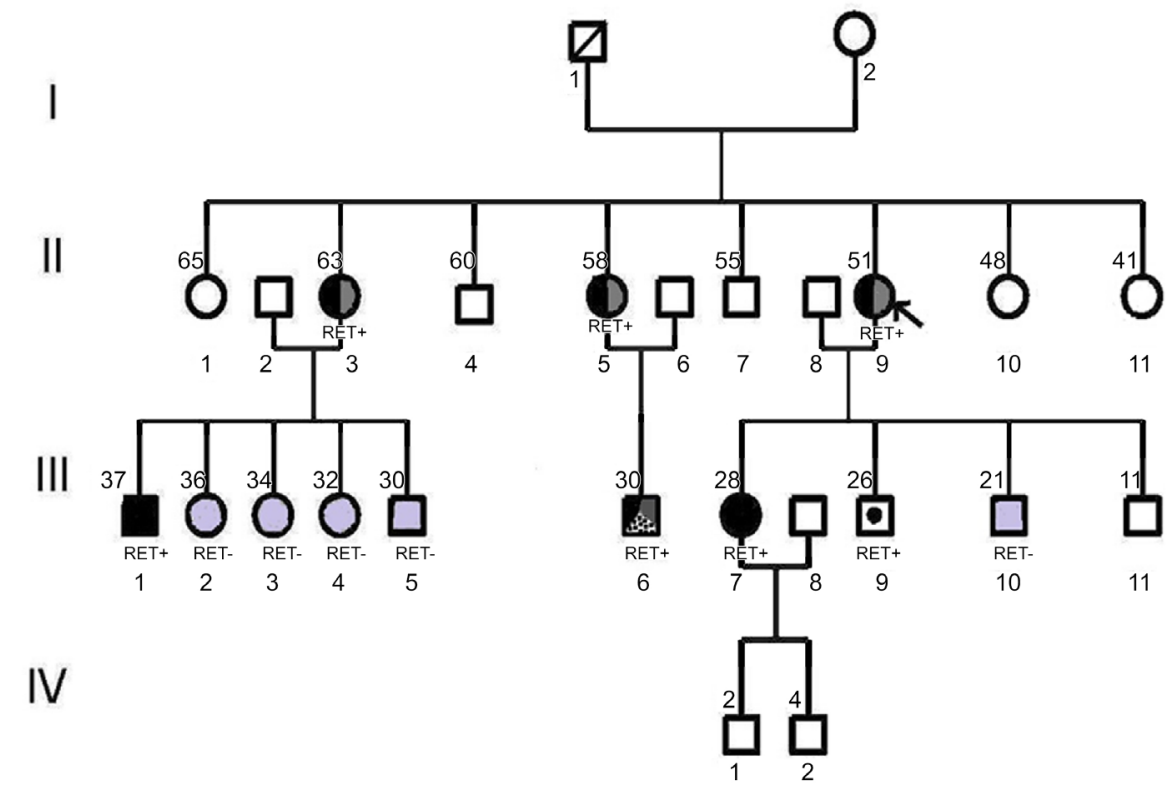

\footnotetext{
$\square$ : Unexplored individual male and female respectively

\Ø: Person died

RET - : Absence mutation

D. Index Case

$R E T+\quad$ : Identified mutation

Mutation holder unexplored

员ET- $\bigcirc_{\text {RET- }}$ : Healthy individual

RET+ at the lesional level

$\square_{\text {RET+ }}$ RET+ $:$ MTC

$\bigcup_{\text {RET+ }}:$ MTC + Primary HPT

: MTC + Primary HPT + Pheochromocytoma

Note: the figures above the symbols indicate the age of the patients

MTC: Medullary thyroid cancer; HPT : hyperparathyroidism
}

Figure 1. Pedigree of second household. 


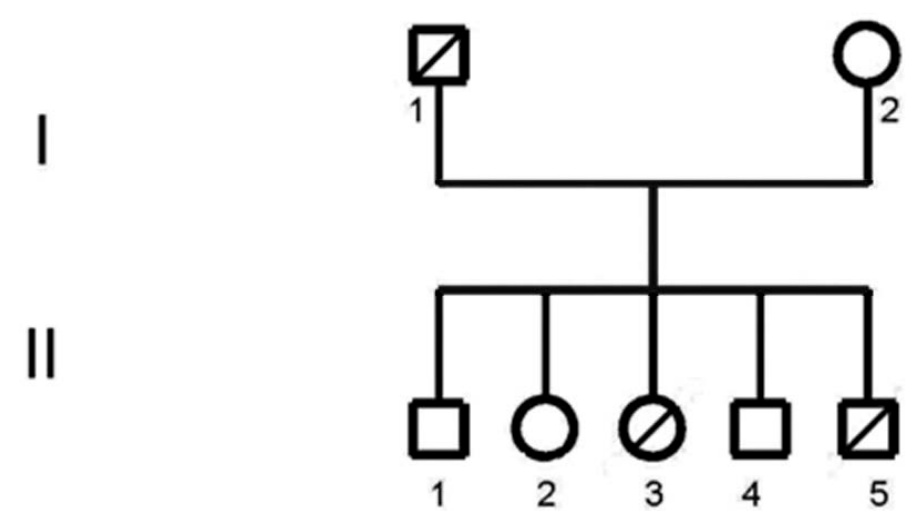

Figure 2. Pedigree of first household.

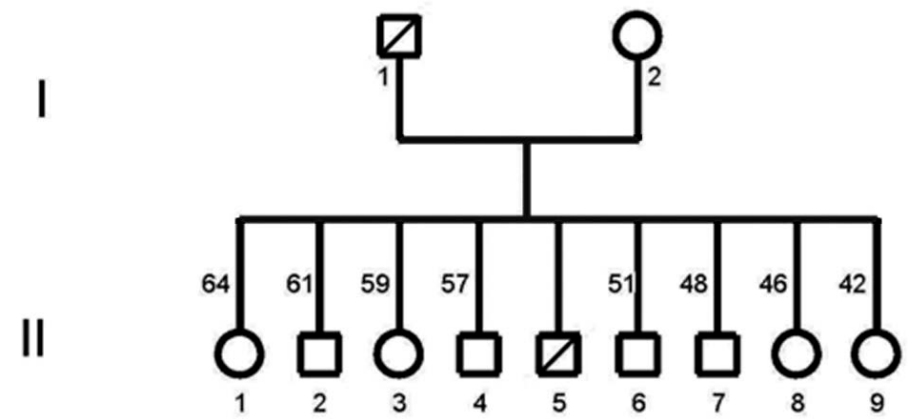

Figure 3. Pedigree of third household.

Table 1. Distribution of patients by age at diagnosis.

\begin{tabular}{ccc}
\hline Age (years) & Number of cases & Frequency (\%) \\
\hline $1-19$ & 3 & 23.1 \\
$20-39$ & 7 & 53.8 \\
$40-59$ & 3 & 23.1 \\
$60-79$ & 0 & 0 \\
\hline
\end{tabular}

\section{Diagnosis of the index case}

The patient (II.9 Figure 2) was sent in 1997 to the Internal Medicine and Nephrology Units of Le Dantec Teaching Hospital to pursue the investigation of Cachi-Ricci disease. This disease was diagnosed at Urology department after recurrent nephritic colic and urinary infection episodes. The diagnosis of primary HPT was carried and parathyroidectomy was indicated. During the surgery, a thyroid nodule was found, which histopathological examination concluded to a MTC. The simultaneous fortuitous discovery of primary HPT and MTC led to a hypothesis of NEM 2A and thus was the circumstance of discovery of the family studied.

\section{Research of other family cases}

After establishing the family tree of the index case, a family survey allowed to diagnose the other cases of MEN 2A in the family. The diagnostic delay of MEN $2 \mathrm{~A}$ on the other family members compared to the index case was on average 7.7 years with extremes of 1 and 14 years (Table 2). 
On 7 of our patients, a well-defined phenotype was identified. Only one patient had MEN 2A1 (pheochromocytoma, primary HPT and MTC). MEN 2A3 (MTC and primary HPT) was recorded on 3 patients (Figure 4).

An "isolated" MTC was noted in 3 patients.

A MTC was recorded on 7 patients, 53.8\%. It was asymptomatic in all cases. Any evocative manifestation of locoregional or distant metastasis was clinically found. Pheochromocytoma was present on only one patient. The latter was asymptomatic. Primary HPT was recorded on four patients, 30.1\%. In three quarters of the cases, it was symptomatic. Renal lithiasis with recurrent unilateral or bilateral nephritic colic attacks was the main manifestation. On the patient II.5 (Figure 2) a notion of urinary calculus emission was found. Others clinical manifestations as articular, neuropsychiatric or cardiac sign were not noted.

Biological diagnosis of MTC was mainly performed by serum calcitonin levels. It was performed on 7 patients and was increased in all cases. On average, it was about $272.6 \mathrm{ng} / \mathrm{L}$ with extremes of 44 and $528 \mathrm{ng} / \mathrm{L}$. Biological research of pheochromocytoma was performed by urinary or plasma methoxyamines. For the primary HPT, the biological research was based on the dosage of total or ionized serum calcium levels, urinary calcium and parathyroid hormone (PTH). Simultaneous increasing of serum PTH and calcium levels or ionized calcium allowed to retain the diagnosis of primary HPT. On average, serum PTH levels were 226.3 $\mathrm{ng} / \mathrm{L}$ with extremes of 70 and $430 \mathrm{ng} / \mathrm{L}$.

Cervical ultrasound found a thyroid nodule on 3 patients running a MTC. Any cervical lymph node impairment was noted on the ultrasound of our patients. Computed tomography (CT) and/or magnetic resonance imaging (MRI) were realized to complement the ultrasound on our patients. Abdominal CT and

Table 2. Diagnostic delay of family members.

\begin{tabular}{ccc}
\hline Diagnostic delay (years) & Number of cases & Frequency (\%) \\
\hline $0-4$ & 3 & 25 \\
$5-9$ & 2 & 16.6 \\
$10-14$ & 7 & 58.4 \\
\hline
\end{tabular}

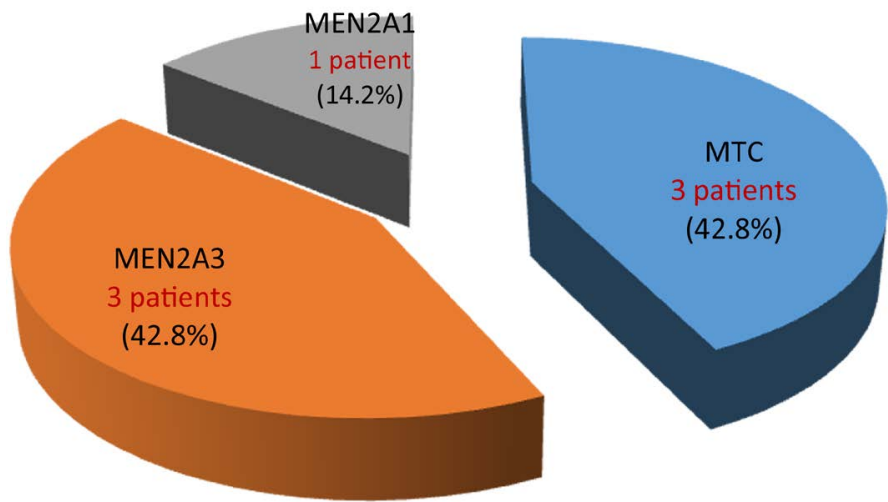

Figure 4. Distribution of patients with MEN 2A phenotype. 
MRI concluded to a normal aspect of adrenal glands on patients with pheochromocytoma. 123I-MIBG scintigraphy was not performed.

On patients with primary HPT, cervical ultrasound showed an evocative aspect of parathyroid gland enlargement on 2 of them and a suggestive aspect of parathyroid adenoma on another one. A normal aspect of parathyroid glands on the ultrasound and MRI was noted on the fourth patient with primary HPT. 99 mTc-Sestamibi scintigraphy was not performed on any patient.

Besides the index case, 11 patients received a genetic testing, $92.3 \%$ of the cases. On 7 patients, proto-oncogene RET mutation was recorded. It was about a punctual mutation on codon 634 for all patients. The only one patient (II.5 Figure 1) who didn't benefit from a genetic testing presented evocative biological signs of MTC with calcitonin levels at 52-fold the normal rate.

\section{Management data}

On the 7 patients running MTC, 3 of them knew a total thyroidectomy with a lymph node cure. Substitutive hormonotherapy with sodium levothyroxine was considered afterwards. Pathological examination of the operative piece confirmed MTC on 2 patients. The other four patients were divided as follow: two patients (III.1 and III.7 Figure 2) are always waiting for a management, one patient (III.6 Figure 2) was lost sight, and one patient (II.5 Figure 2) died.

The patient (III.6 Figure 2) who was running pheochromocytoma was lost sight. A selective resection of parathyroid glands was realized on three patients among those with primary HPT.

Pathological examination of resected parathyroid glands concluded to two cases of diffuse hyperplasia and a case of adenoma. A lost sight was recorded among them (III.6 Figure 2)

Prophylactic thyroidectomy was not performed on any of our patients.

\section{Evolving data}

On three operated patients of MTC, we had the evolving data of two patients. On the patient II.5 (Figure 2) the outcome was favourable with a stable serum calcitonin levels at less than $2 \mathrm{ng} / \mathrm{L}$ after a regress of 4 years. Regarding the index case, biological and radiological monitoring found a loco regional residual disease that indicated a surgical revision. Then it was noted the persistence of a thyroid stump associated with cervical lymphadenopathy that leads to a third surgical revision that considers a bilateral lymph node cure. Cervical radiotherapy was also performed. After a regress of 5 years, serum calcitonin levels remained elevated. However, the PET-Scan of the whole body doesn't find a residual disease.

\section{Discussion}

We reported in this work the first Senegalese family with documented MEN 2A. It's about a large family of three households with 22 brothers, sisters, step-brothers, step-sisters, and 12 children and grand-children. It can be compared to the one reported by Benazzouz et al. down Marocco [5]. The average age of 31.5 years at 
the moment of the diagnosis of ours patients and female predominance recorded in this study is similar to most family with MEN 2A reported in the literature [5] [6] [7]. In the family, only 13 people (index case included) were probed. The absence of monitoring register of pathologies with genetic predisposition in our structures, the cost of genetic investigations bear by the patients themselves, the unavailability of genetic tests and some traditional considerations are all factors that may explain this low number.

The simultaneous discovery of primary HPT and MTC constituted the circumstance of discovery of MEN 2A on our index case. A similar case was reported in Japan but contrary to our patient, that's the surgical exploration that led to the intraoperative casual discovery of a parathyroid adenoma [8]. MTC was the circumstance of discovery of MEN 2A mostly found [5]. The discovery of MEN 2A on an index case imposes systematically, because of the family character of the disease, the exploration of the family members (siblings and direct relatives) after informed consent [9] [10].

And that's what was done in this case with a family survey and the working out of the family tree that allowed to diagnose the other cases of MEN $2 \mathrm{~A}$ in the family.

In this family, a MTC was recorded on 7 patients and it was asymptomatic, in all cases. Hence the importance of systematic screening in families at risk. This screening was performed on our patients by serum calcitonin levels dosage. In our series, no patient had a complete extension radiological assessment, whereas it was necessary for most of them.

The only one case of documented pheochromocytoma in this family was also asymptomatic. Pheochromocytoma is symptomatic in only one third of cases during MEN 2A [11]. CT and MRI did not show a tumor. The MIBG scintigraphy, which has a better sensitivity and specificity than these examinations for the tumor localization during pheochromocytoma, was not realized in our patient because of its unavailability in our country [9].

Genetic testing for a mutation of the proto-oncogene RET was carried out in 12 individuals, that to say $92.3 \%$ of the cases. In more than half of the cases (58.3\%), a mutation of the proto-oncogene RET was found. This result is similar to the one from Benazzouz et al. [5] who found the mutation in $53.8 \%$ of detected individuals among the family members they report. The mutation found in our patients was the same. It was codon 634 of exon11. Codon 634 mutations in exon 11 are the most common during MEN 2A [9]. They are found in several populations [5] [6] [7]. This mutation leads to the substitution of a cysteine by a tyrosine (C634Y). The presence of this mutation is associated with a high risk of MTC [9]. That seems to be proved by our results with the presence of a MTC on our patients of this family presenting this mutation subject to a single patient who didn't receive clinical, biological and radiological investigations. In the contrary, cutaneous manifestations during MEN 2A like lichen amyloid that might be more common on individuals with a mutation of codon 634 were not rec- 
orded in our patients [12].

For a curative purpose, surgery was the main treatment indicated in our patients. It involved 3 patients with MTC and 3 individuals with primary HPT. It was noted three patients lost sight. Two patients are still waiting for surgery and another one has died before surgery. These data demonstrate insufficiencies of care that can be explained in our context by a lack of technical facilities and the cost of this care that is most often bear by patients themselves. The histopathological examination confirmed the MTC in two of our operated patients. For patients with primary HPT and who are operated, histology concluded to diffuse hyperplasia in two cases and adenoma in one case.

The postoperative issues were varied in patients with CMT. For one, the evolution is favourable so far with a calcitonin level of less than $2 \mathrm{ng} / \mathrm{L}$. On the other hand, in the index case, the evolution was marked by a locoregional residual disease that required several surgical revisions and cervical radiotherapy. The calcitonin levels remain high despite the absence of lesions in the various radiological examinations including PET-SCAN. A similar evolution is noted in the index case of a Moroccan family of MEN 2A but contrary to our patient, bone and liver metastases were found on imaging [5].

\section{Conclusion}

In the context of the MEN 2A, genetic investigation to research the mutation of the proto-oncogene RET is a capital moment for the development of discriminative strategies of preventive and curative management of the adrenal, thyroid and parathyroid tumors. Thus, systematic screening of family members may reveal other cases. But we, in developing countries, are facing defect of powerful technical support centre and financial scanty means of our patients that limit exploration of the disease deeply.

\section{References}

[1] Leye, A., Ndiaye, M., Bourdin, C., Leye, Y., Gueye, S., Ndiaye, N., Deguenonvo, R., Calender, A. and Moreira-Diop, T. (2009) Multiple Endocrine Neoplasia Type 2A: Diagnostic and Therapeutic Management of One Case. Dakar Médical, 54, 78-84.

[2] Bauters, C., Leclerc, I., Wemeau, J.-L., Proye, C., Pigny, P. and Porchet, N. (2003) Multiple Endocrine Neoplasia. Recent Advances in Clinical and Genetic Diagnosis. La Revue de Médecine Interne, 24, 721-729. https://doi.org/10.1016/S0248-8663(03)00212-1

[3] Sipple, J.H. (1961) The Association of Pheochromocytoma with Carcinoma of the Thyroid. American Journal of Medicine, 31, 163-166. https://doi.org/10.1016/0002-9343(61)90234-0

[4] Diouf, B., Dia, D., Ka, M.M., et al. (1999) Description of a Case of Cacchi Ricci Disease Associated with Hyperparathyroidism in the Setting of Multiple Endocrine Disease. Dakar Médical, 44, 229-231

[5] Benazzouz, B., Hafidi, A., Benkhira, S., Chraibi, A., Kadiri, A. and Hilal, L. (2008) C634R Mutation of the Proto-Oncongene RET and Molecular Diagnosis in Multiple Endocrine Neoplasia Type 2 in a Large Moroccan Family. Bulletin du Cancer, 95, 
457-463.

[6] Punales, M.K., Graf, H., Gross, J.L. and Maia, A.L. (2003) RET Codon 634 Mutations in Multiple Endocrine Neoplasia Type 2: Variable Clinical Features and Clinical Outcome. Journal of Clinical Endocrinology and Metabolism, 88, 2644-2649. https://doi.org/10.1210/jc.2002-021422

[7] Benazzouz, B., Chraïbi, A., Doghmi, Y., El Bacha, S., Boutayeb, S. and Kadiri, A. (2006) Characterization of RET Proto-Oncogene C634Y Mutation in a Moroccan Family with Multiple Endocrine Neoplasia Type 2A. Annales d'Endocrinologie, 67, 21-26. https://doi.org/10.1016/S0003-4266(06)72535-5

[8] Yonekawa, H., Sugitani, I., Fugimoto, Y., Arai, M. and Yamamoto, N. (2007) A Family of Multiple Endocrine Neoplasia Type 2A (MEN 2A) with Cys 630 Tyr RET Germline Mutation Report a Case. Endocrine Journal, 54, 531-535.

https://doi.org/10.1507/endocrj.K06-145

[9] Niccoli-Sire, P. and Conte-Devolx, B. (2007) Multiple Endocrine Neoplasia Type 2. Annales d'Endocrinologie, 68, 317-324. https://doi.org/10.1016/j.ando.2007.04.005

[10] Petr, E.J. and Else, T. (2016) Genetic Predisposition to Endocrine Tumors: Diagnosis, Surveillance and Challenges in Care. Seminars in Oncology, 43, 582-590. https://doi.org/10.1053/j.seminoncol.2016.08.007

[11] Conte-Devolx, B., Niccoli-Sire, P. and Endocrine Tumor Study Group (2010) Clinical Characteristics of Multiple Endocrine Neoplasia. Bulletin de l'Academie Nationale de Medecine, 194, 69-79.

[12] Verga, U., Fugazzola, L., Cambiaghi, S., et al. (2003) Frequent Association between MEN 2A and Cutaneous Lichen Amyloidosis. Clinical Endocrinology, 59, 156-161. https://doi.org/10.1046/j.1365-2265.2003.01782.x 